Case Report

\title{
Abdominal Distension Associated with Luminal Fungi in the Intestines of Axolotl Larvae
}

\author{
Chiara Zullian, ${ }^{1}$ Aurore Dodelet-Devillers, ${ }^{1}$ Stéphane Roy, ${ }^{2}$ \\ Pierre Hélie, ${ }^{3}$ and Pascal Vachon ${ }^{1}$ \\ ${ }^{1}$ Département de Biomédecine Vétérinaire, Faculté de Médecine Vétérinaire, Université de Montréal, \\ Saint-Hyacinthe, QC, Canada J2S 2M2 \\ ${ }^{2}$ Département de Stomatologie, Faculté de Médecine Dentaire, Université de Montréal, Montréal, QC, Canada H3C 3J7 \\ ${ }^{3}$ Département de Pathologie et Microbiologie, Faculté de Médecine Vétérinaire, Université de Montréal, \\ Saint-Hyacinthe, QC, Canada J2S 2M2
}

Correspondence should be addressed to Chiara Zullian; chiara.zullian@nemo.unipr.it

Received 20 March 2015; Accepted 5 May 2015

Academic Editor: Carlos Gutierrez

Copyright (C) 2015 Chiara Zullian et al. This is an open access article distributed under the Creative Commons Attribution License, which permits unrestricted use, distribution, and reproduction in any medium, provided the original work is properly cited.

Axolotls show a remarkable regeneration capacity compared with higher vertebrates, regenerating missing appendages such as limbs and tail as well as other body parts (i.e., apex of the heart, forebrain, and jaw) after amputations which makes this animal a very interesting research model for tissue regeneration mechanisms. Larvae are individually housed in a $20 \%$ Holtfreter's solution within clear plastic containers. The photoperiod light: darkness cycle is $12: 12 \mathrm{~h}$. Larvae with a total body length of less than $5 \mathrm{~cm}$ are fed once a day with large brine shrimp and blood worm. Albino larvae appeared to have a tendency to exhibit abdominal distention. No clinical signs of illness seemed to be associated with the condition; however, these animals exhibit a relatively slower growth rate. To better characterize this condition, we performed histological sectioning for cross sectional slide preparation on wild type and albino axolotl larvae following euthanasia. The only lesion seen in the albino larvae was a thickened gut wall and the presence of fungi within the intestines. We hypothesize that this may be due to a lower efficacy of the albino larvae's immune system.

\section{Introduction}

The Mexican axolotl (Ambystoma mexicanum) is a urodele amphibian of the family of Ambystomatidae. The axolotl is an inducible obligate neoteny, remaining in the water-dwelling larval state for its entire life, unless being artificially induced to mature [1].

As other urodele amphibians, axolotls show a remarkable regeneration capacity compared with higher vertebrates. They can regenerate missing appendages after amputation such as limbs and tail as well as regenerating other body parts like the apex of the heart, the forebrain, and sections of their jaw [2]. Thanks to this unique feature, these animals represent a widespread research model in the field of embryology, aging, and regenerative medicine and hopefully, in the future, we will achieve more knowledge from urodele amphibian's physiology, which will help to find applications to organ damage in higher vertebrates.
Axolotls are usually vigorous animals. When their health does fail, illness may become a serious problem that can be attributed to different causes, most frequently identified as noninfectious and infectious. The first and most important cause of poor health is nonoptimal environmental conditions while the other less frequent problems are associated with bacterial infections and parasitic infestations [3].

Noninfectious causes of disease such as poor water quality and other inappropriate conditions of husbandry (nutrition, water temperature control, and environmental stressors) can be direct causes of distress and death and they can be a source of chronic stress leading to secondary infections by opportunistic pathogens such as Actinomycetes, Aeromonas, Salmonella, Pseudomonas, Acinetobacter, Proteus, and Alcaligenes [4].

If adults are relatively easy to maintain; however, young larvae seem to be very vulnerable to disease and difficult to treat. The most effective strategy to avoid morbidity and 


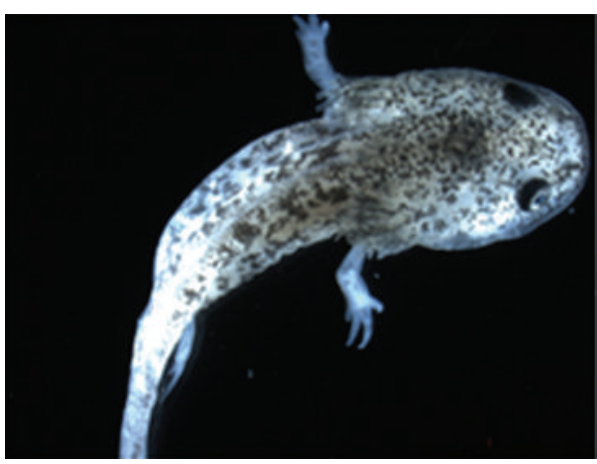

(a)

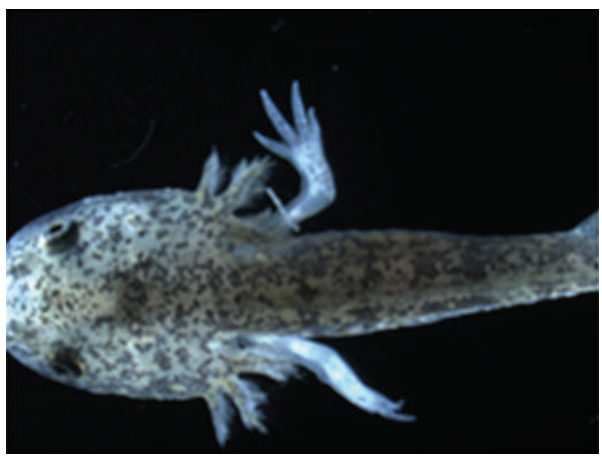

(c)

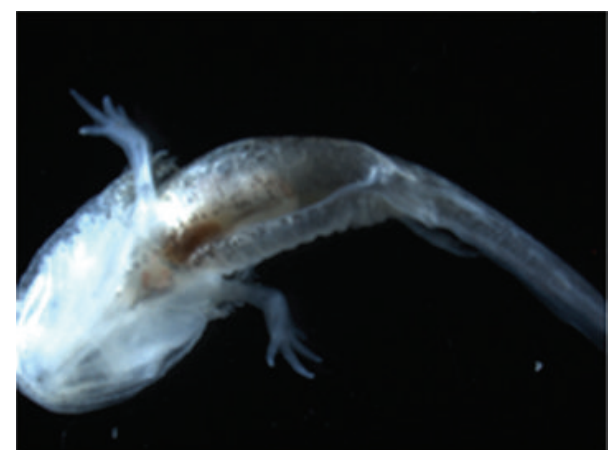

(b)

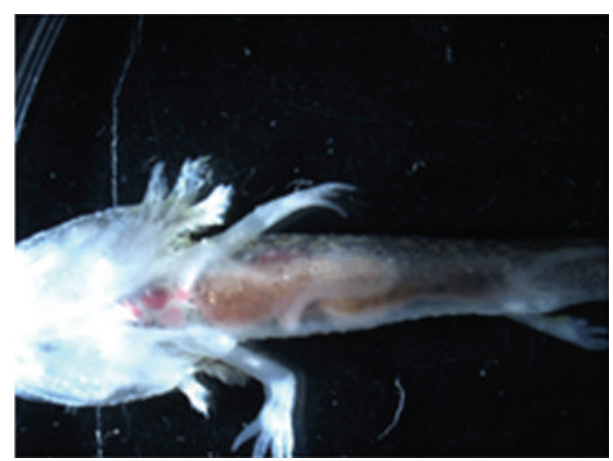

(d)

FIGURE 1: Dorsal and ventral views of axolotl larva with abdominal distension ((a) and (b)). Dorsal and ventral views of a normal axolotl larva $((c)$ and $(d))$. Note the presence of abdominal fluid in the larva with the distension (b).

mortality is based on feeding them generously, paying strict attention to water quality and avoiding overcrowding [5].

\section{Case Presentation}

Axolotl larvae are maintained in a rearing room at the University of Montreal, in the Department of Stomatology, Faculty of Dentistry. Animals in this colony are purchased from the Ambystoma Genetic Center Stock (Lexington, KY, USA) and measure between $2.0 \mathrm{~cm}$ to $8.5 \mathrm{~cm}$ from the snout to tip of the tail, at arrival. Larvae are individually housed within clear plastic containers filled with a $20 \%$ Holtfreter's solution (a buffered ( $\mathrm{pH}$ 7.0) carbon filtered deionized water salt solution) at a temperature varying from 20 to $23^{\circ} \mathrm{C}$. The photoperiod is a $12: 12$ (light: dark) cycle. Larvae with body length of less than $5 \mathrm{~cm}$ are fed once a day with large brine shrimp and blood worm (Hikari Aquatic Diets, Hayward, CA, USA), while those measuring more than $5 \mathrm{~cm}$ are fed 3 times a week with the same products. Animals with a total body length of $9 \mathrm{~cm}$ are considered juveniles, and when longer, they are considered adults. They are both housed in simple aquariums in which water treatment consists of purification through a carbon filter system only.

We have observed that mainly albino axolotl larvae (less than $3 \mathrm{~cm}$ ) may show abdominal distension for which no previous cause has been identified (Figure 1). No apparent clinical signs of illness or discomfort seemed to be associated with the condition. It has been reported that these larvae could not reach a body length of more than $4-5 \mathrm{~cm}$ and therefore would not attain maturity. They were then discarded as they could not be used for the experimental purposes by the researchers.

Very little information is available about axolotl's diseases, as the published literature on this species is very scarce. To better understand the possible cause of abdominal distension in these animals, we performed histopathology on axolotls larvae (10 weeks old) affected by this condition. We also assessed the normal histological aspect in healthy wild type larvae of the same age. Euthanasia was performed by placing animals in a $0.2 \%$ solution of MS-222 (Tricaine Methanesulfonate) buffered solution for a period of $20 \mathrm{~min}$. Fixation of the entire body was thereafter conducted with a solution of formaldehyde $10 \%$. Larvae were embedded in paraffin, and longitudinal or cross sections (10 $\mu \mathrm{m}$ thick) were performed using a microtome and subsequently stained with hematoxylin and eosin. Figure 2 shows a cross section of a normal wild type axolotl. No apparent sign of infection, or lesion, was observed in these animals when looking at all organs.

Interestingly, the histological sections of the affected larvae showed the presence of fungi in the lumen of the intestine (Figure 3). What can also be appreciated is the clear appearance of the gut wall and an increased thickness compared to the healthy intestines. The reason for this condition has not been established; however, we hypothesize that this 


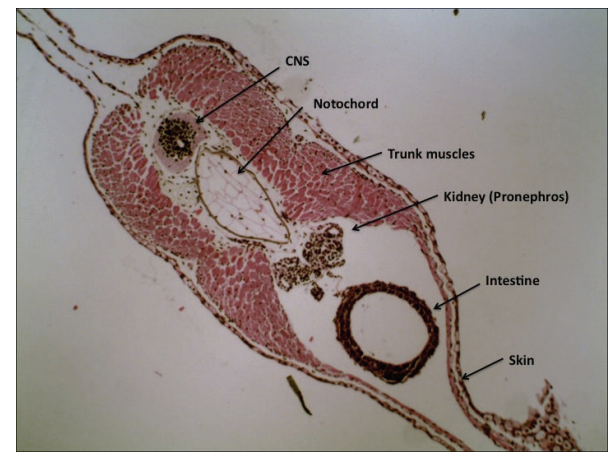

FIGURE 2: Histological cross section of the body of a healthy 10week-old larva axolotl (H\&E stain, 10x). Note the clear appearance of the intestinal lumen and compactness of the gut wall.

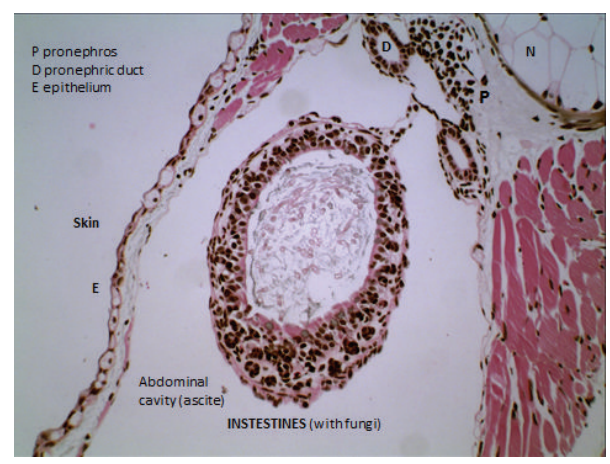

FIGURE 3: Histological section showing the intestine of an infected axolotl larva (10 weeks old). Note the presence of fungi in the intestinal lumen and the thickening of the intestinal wall (H\&E stain, 10x).

may be due to a lower efficacy of the albino larvae's immune system, considering that in the wild type larvae the condition has not been observed nearly as frequently and that the albino axolotls are known to have some immunodeficiency.

\section{Discussion}

This is the first case report of abdominal distention in axolotl larvae showing the abnormal presence of fungi in the intestinal lumen. It would have been interesting to verify if fungi may be responsible for the release of chemical factors that could induce a change in vessel permeability or intestinal cell functions and subsequently initiate this condition in the affected subjects. In humans, abdominal bloat is sometimes associated with fungi [6] such as Candida spp. which are known to produce many toxins [7] that may alter normal physiological functions [8]. For the future, it would be of interest to characterize the fungi to better understand the pathology and possibly to eradicate or minimize this condition through antifungal treatments.

\section{Conflict of Interests}

The authors declare there is no conflict of interests in the publication of this case report.

\section{Acknowledgments}

The authors wish to thank Dr. Stéphane Roy (Département de Stomatologie, Université de Montréal) for giving them access to the axolotl larvae. Histopathological costs were supported by the Fond de Développement en Médecine des Animaux de Laboratoire (Pascal Vachon).

\section{References}

[1] C. C. Martin and R. Gordon, "Differentiation trees, a junk DNA molecular clock, and the evolution of neoteny in salamanders," Journal of Evolutionary Biology, vol. 8, no. 3, pp. 339-354, 1995.

[2] M. Lévesque, É. Villiard, and S. Roy, "Skin wound healing in axolotls: a scarless process," Journal of Experimental Zoology Part B: Molecular and Developmental Evolution, vol. 314, no. 8, pp. 684-697, 2010.

[3] P. W. Scott, Axolotl: Care and Breeding in Captivity, Tropical Fish Hobbyist Publications, Neptune City, NJ, USA, 1995.

[4] S. T. Duhon, "Diseases of axolotls," in Developmental Biology of the Axolotl, J. B. Armstrong and G. M. Malacinski, Eds., pp. 264269, Oxford University Press, New York, NY, USA, 1989.

[5] J. B. Armstrong, S. T. Duhon, and G. M. Malacinski, "Raising the axolotl in captivity," in Developmental Biology of the Axolotl, J. B. Armstrong and G. M. Malacinski, Eds., pp. 220-227, Oxford University Press, New York, NY, USA, 1989.

[6] A. Erdogan and S. S. Rao, "Small intestinal fungal overgrowth," Current Gastroenterology Reports, vol. 17, no. 4, 2015.

[7] F. L. Mayer, D. Wilson, and B. Hube, "Candida albicans pathogenicity mechanisms," Virulence, vol. 4, no. 2, pp. 119-128, 2013.

[8] M. Polke, B. Hube, and I. D. Jacobsen, "Candida Survival Strategies," in Advances in Applied Microbiology, vol. 91 of Advances in Applied Microbiology, pp. 139-235, Elsevier, 2015. 

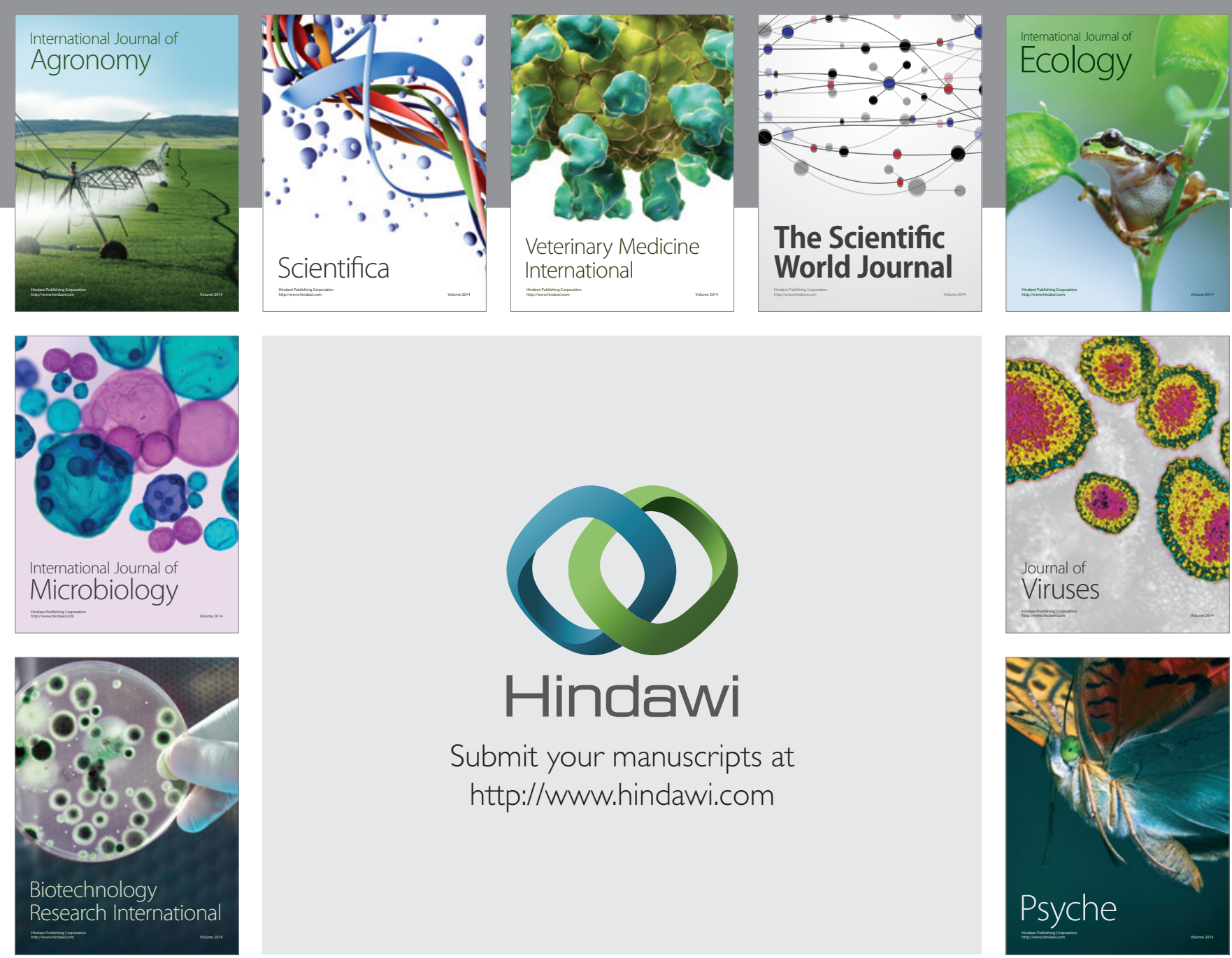

Submit your manuscripts at http://www.hindawi.com
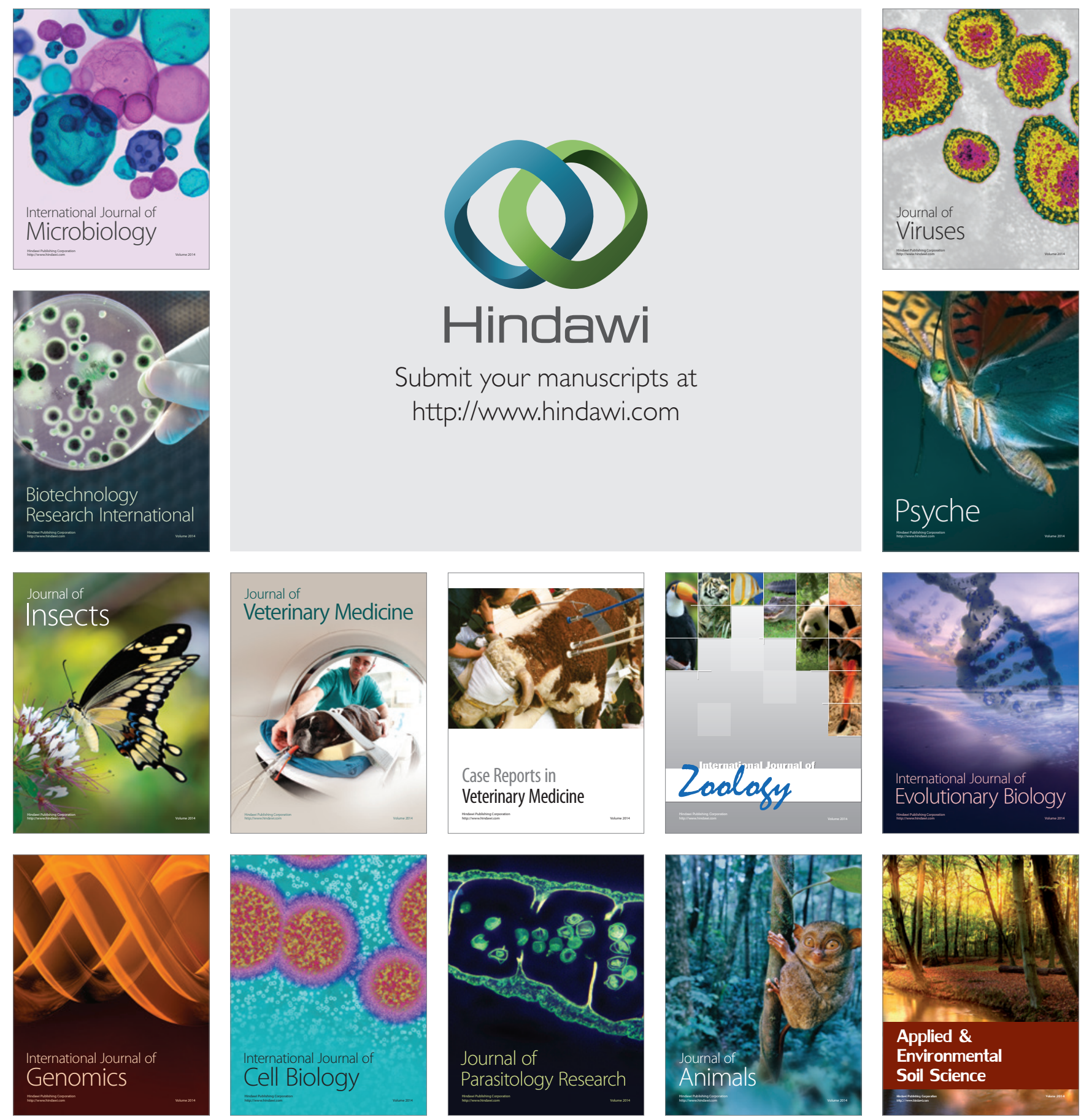